\title{
A New Post-column Energy-filter Design For Conventional S/TEMs
}

\author{
C. G. Trevor, M. M. G. Barfels, D. Moonen, R. Nita, H. Chao, R. Harmon, M. K. Kundmann H.A. \\ Brink and J.A. Hunt
}

Gatan Research and Development, 5933 Coronado Lane, Pleasanton CA 94588

Post-column imaging filters on TEMs are powerful analytical tools for the purpose of elemental and chemical mapping, image resolution and contrast enhancement through zero-loss filtering [1], high resolution quantitative analysis through EELS spectroscopy and EELS spectrum imaging [2].

We present a new post-column filter designed for TEMs up to $400 \mathrm{keV}$ equipped with an electron source of energy spread greater than $0.3 \mathrm{eV}$. The chief advantages of the new design include a superior high-speed, high-resolution $2 \mathrm{k}$ x 2k CCD imaging system and improved electron optics which simplify the energy-filter operation and increase the field of view by a factor of almost 3 .

Improved electron optics allow for a reduction in the imaging filter (or post-TEM) magnification, which increases both the field of view and simplifies EFTEM operation. The prism aberration correction has been tailored to match the energy spread of the electron source by constraining the maximum isochromatic error to be less than $1.5 \mathrm{eV}$ through a $5 \mathrm{~mm}$ filter entrance aperture. Figure 1 shows a comparison of the isochromatic figure of the GIF 2000 and the new filter. The maximum geometric distortion has been kept below 1.5\% (see Figure 2), and the system achromaticity results in a smearing of less than one pixel.

The new imaging system is based on the UltraScan 1000[3], a 2k x 2k, 14 micron pixel, 4-port readout camera. For use on the filter this camera was modified to double the frame rate to approximately 10 frames per second. This allows a 'live' view suitable for scanning the sample and focusing the image. By comparison with a TV camera this new view mode has a number of advantages; it has better contrast, it is more sensitive and it is in the same plane as the high quality detector. Removing the TV camera also removes a potential source of noise and simplifies the use of the system. The 'High-Contrast-Resolution' optics used on the camera have a much improved point spread function over previous generation cameras. This improves the quality of both images and spectra, (as previously shown with our dedicated spectrometer, the 'Enfina' [3]).

The first such filter was installed at McMaster University, Ontario, Canada on a JEOL 2010F. Figures 1 and 2 show the isochromatic figure and geometric distortion as seen on this system. The spectral energy resolution (at very low emission) was measured to be $0.55 \mathrm{eV}$ in 0.1 second, Figure 3 . Noise analysis from a time resolved CCD readout mode showed a maximum movement of about $50 \mathrm{meV}$ from fields and other noise sources at $60 \mathrm{~Hz}$ and above.

The combination of improved optics, upgraded detector system, and better software gives the new energy filter a significant boost in performance and ease of use compared to its predecessors.

[1] R. F. Egerton, Electron Energy-Loss Spectroscopy in the Electron Microscope, Plenum Press [2] J. A. Hunt and D.B. Williams, Ultramicroscopy 38 (1991) 47-73

[3] H. A. Brink et al, Proceedings of Microscopy and Microanalysis 2000, 212-213 

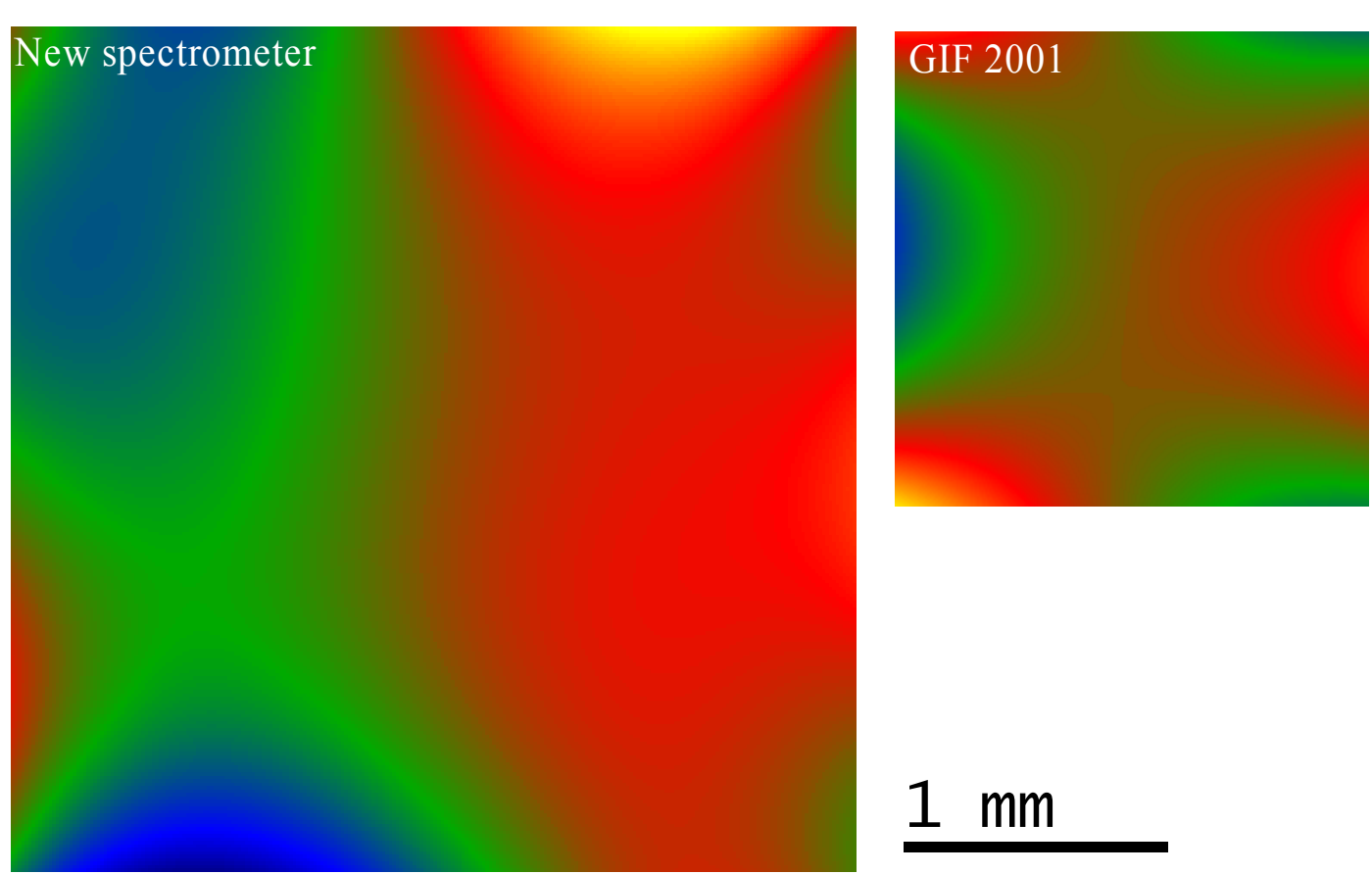

\section{$1 \mathrm{~mm}$}

Figure 1: Comparison of isochromacity of new spectrometer and GIF 2001

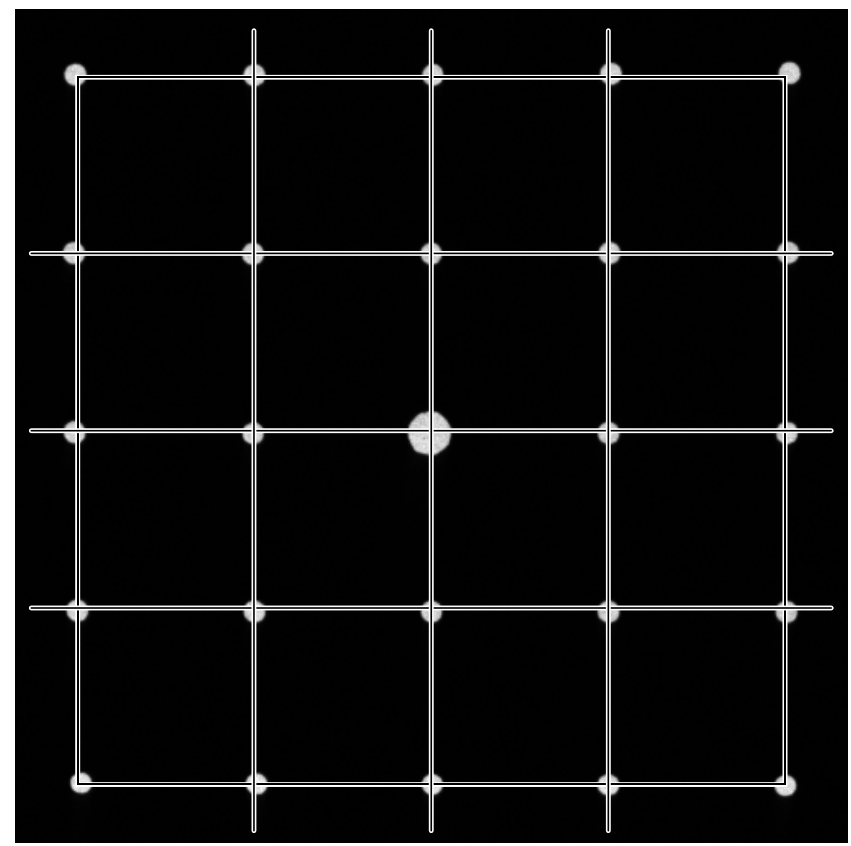

Figure 2: Geometric distortion in image $(<1.5 \%)$

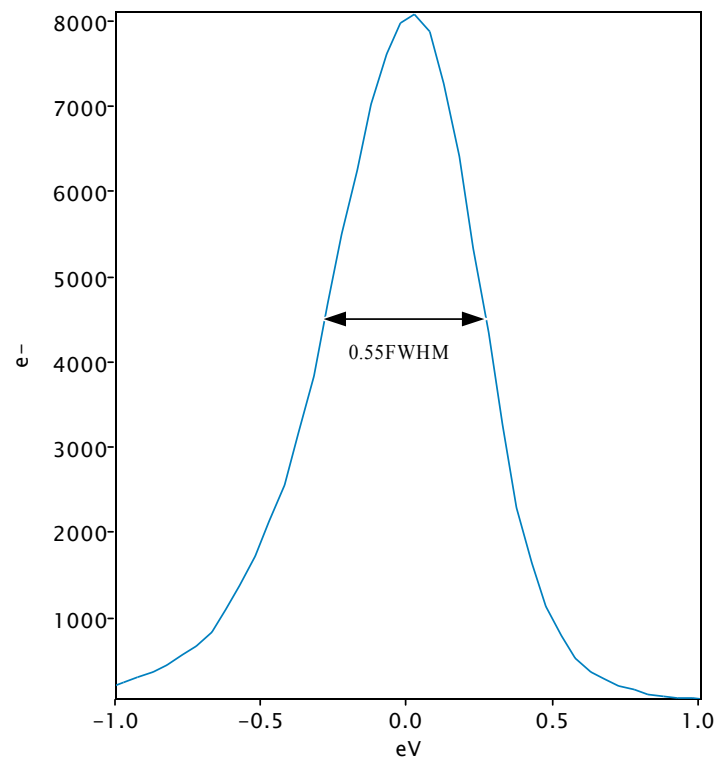

Figure 3: Energy resolution at low emission current in 0.1 seconds 